\title{
AUDITORIA DA OUALIDADE DOS REGISTROS DE ENFERMAGEM EM PRONTUÁRIOS EM UM HOSPITAL UNIVERSITÁRIO
}

Valdenir Almeida da Silva1 ${ }^{1}$ Rosana Santos Mota1 ${ }^{1}$ Larissa Silva Oliveira, Nilcea de Jesus², Cristiane Marques de Carvalho¹, Livia Gomes da Silva Magalhães ${ }^{1}$

Objetivo: Analisar a qualidade dos registros de enfermagem em prontuários. Metodologia: Pesquisa descritiva, quantitativa realizada em um hospital universitário de Salvador, Bahia, Brasil, entre setembro de 2017 e maio de 2018, a partir de indicadores de qualidade dos registros de enfermagem em prontuários. Utilizou-se um instrumento estruturado para a coleta dos dados. A análise foi do tipo descritiva; utilizou-se a frequência absoluta e relativa dos indicadores. Resultados: Dentre os 203 prontuários analisados, o percentual de identificação correta dos pacientes, com nomes completos e número de prontuário foi acima de $90 \%$. Houve fragilidade na checagem das prescrições médica e de enfermagem, assim como na justificativa para a não checagem de itens prescritos. Conclusão: A qualidade dos registros de enfermagem atende parcialmente às recomendações do Conselho Federal de Enfermagem. Os resultados sugerem necessidade de constante atuação dos programas educacionais para o fortalecimento de ações que visem a excelência dos registros de enfermagem.

Descritores: Auditoria de Enfermagem; Qualidade da Assistência à Saúde; Registros de Enfermagem Educação Permanente.

\section{QUALITY AUDIT OF NURSING REGISTERS ON PATIENTS' RECORDS IN A UNIVERSITY HOSPITAL}

Objective: To analyze the quality of the nursing records on patients' records. Methodology: A descriptive study of a quantitative approach performed in a university hospital of Salvador, Bahia, Brazil, between the months of September 2017 and May 2018 , from quality indicators of the nursing registers on patients' records. A structured instrument was used for data collection. The analysis was descriptive; it was used the absolute and relative frequency of the indicators. Result: Among the 203 patients' records analyzed, the percentage of correct identification of patients, with full name and number of the clinical record was of over $90 \%$. Nevertheless, there was a weakness in checking medical and nursing prescriptions, as well as in the justification for not checking prescribed items. Conclusion: The quality of the nursing registers partially follows the recommendations of the Federal Council of Nursing. The results suggest the need for constant practice of educational programs for strengthening actions towards performing nursing registers with excellency.

Descriptors: Nursing care audit; Quality in Healthcare; Nursing Registers; Permanent Education.

\section{AUDITORIA DE CALIDAD DE REGISTROS DE ENFERMERİA EN HISTORIALES CLÍNICOS DE UN HOSPITAL UNIVERSITARIO}

Objetivo: Analizar la calidad de registros de enfermería en historiales. Metodología: Pesquisa descriptiva, con abordaje cualitativa realizada en un hospital universitario de Salvador, Bahia, Brasil, entre los meses de septiembre de 2017 y mayo de 2018, a partir de indicadores de cualidad de los registros de enfermería en historiales clínicos. Se utilizó un instrumento estructurado para la colecta de datos.

El análisis fue descriptivo; fue usado la frecuencia absoluta y relativa de los indicadores Resultados: De entre los 203 historiales analizados, el porcentaje de identificación correcta de los pacientes, con nombres completos y número de historial fue superior a 90\%. Sin embargo, hubo debilidad en el chequeo de las prescripciones médicas y de enfermería, al igual que en la justificativa para el no chequeo de ítems prescritos. Conclusión: La calidad de los registros de enfermería atiende parcialmente a las recomendaciones del Consejo Federal de Enfermería. Los resultados sugieren una necesidad de constante actuación de los programas educacionales para el fortalecimiento de acciones que tengan por objeto la realización de registro de enfermería con excelencia.

Descriptores: Auditoria de Enfermería; Cualidad de la Atención a la Salud; Registros de Enfermería; Educación Permanente. 


\section{INTRODUÇÃO}

A palavra auditoria tem sua origem no verbo latino audire, que significa ouvir, principio da palavra auditor (do latim auditore - aquele que ouve). Contudo, o termo na língua inglesa - audit imprime mais sentido ao seu objeto por envolver o exame, a correção e a certificação. Historicamente, os auditores eram pessoas designadas a tirarem conclusões fundamentadas em informações verbais que lhes eram transmitidas. Considera-se que esta seja uma função de grande relevância, pois o auditor pode ser o responsável pelas decisões norteadoras do processo operacional, de trabalho e de gestão, a partir de suas conclusões. Para a auditoria, utilizam-se de documentos contendo informações, registros e descrições sobre o processo de trabalho, para o alcance de conclusões fidedignas ${ }^{(1)}$. Assim, a auditoria é caracterizada por critérios para avaliação e revisão dos processos e seus resultados, baseados em aspectos legais, éticos e técnicos. Tais critérios podem ser estabelecidos pela instituição, ou por normas regulamentadoras que norteiam a busca pela qualidade $^{(2)}$

Apesar de a auditoria ser um ramo da contabilidade, diversas áreas profissionais a tem utilizado como uma ferramenta importante para mensurar a qualidade dos serviços e processos de trabalho, a exemplo da enfermagem. A auditoria em enfermagem pode ser definida como avaliação sistemática da qualidade do processo de trabalho da equipe, dentro do cuidado ao paciente. Utiliza-se da verificação dos registros de enfermagem em prontuários como documentação legal, tanto para aspectos administrativos, quanto financeiros. Esse processo é fundamental para a criação de um modelo processual de enfermagem com qualidade a partir de um planejamento, implementação e avaliação de situações-problema dos pacientes, com o objetivo final de prevenir o resolvê-las com equidade $e$ integralidade ${ }^{(3,4)}$. Para o desenvolvimento dos registros de enfermagem, é fundamental conhecer e obedecer as regras estabelecidas pelo Conselho Federal de Enfermagem $(C O F E N)^{(5)}$. Tais regras incluem a utilização de letras legíveis; ser completos; ser realizado com clareza e coerência; informar data e hora do cuidado prestado; conter orientações fornecidas e informações obtidas, dentre outras, que podem ser tomadas como indicadores de qualidade.

Segundo o COFEN, ${ }^{(5)}$ os registros de enfermagem constituem uma forma de comunicação escrita entre os membros do grupo de saúde, de modo a facilitar a coordenação e a continuidade do planejamento, por meio da descrição de procedimentos e ações realizados pelo profissional de enfermagem. Nesses registros, há informações de relevância para a assistência ao indivíduo. A Resolução № 0564/2017 (Código de Ética dos Profissionais de Enfermagem),(6:5) no artigo 36, afirma o dever de "Registrar no prontuário e em outros documentos as informações inerentes e indispensáveis ao processo de cuidar de forma clara, objetiva, cronológica, legivel, completa e sem rasuras". O profissional de enfermagem deve registrar seus escritos conforme o recomendado, para possibilitar um monitoramento e continuidade da assistência com qualidade. A avaliação desses registros favorece a identificação de possiveis deficiências da equipe no processo de trabalho e na documentação da assistência prestada. Tais registros se constituem como ferramenta importante para a revisão detalhada do cuidado prestado, de seus resultados e para subsidiar medidas de aprimoramento.

Diante do panorama sobre a necessidade dos registros de enfermagem para a garantia da qualidade e continuidade da assistência; da importância do fluxo de comunicação entre a enfermagem e a equipe multiprofissional; e da certeza de que os registros corretos, fidedignos e válidos se constituem como um instrumento de segurança para o paciente e para os profissionais, o presente artigo tem como objetivo analisar a qualidade dos registros de enfermagem em prontuários.

\section{METODOLOGIA}

\section{Tipo de estudo}

Trata-se de uma pesquisa descritiva, com abordagem quantitativa.

\section{Local do estudo}

A pesquisa foi realizada em um hospital universitário (HU), pertencente ao Sistema Único de Saúde (SUS), localizado em Salvador, Bahia, Brasil. Trata-se de um hospital de ensino, público, geral, de grande porte, referência em média e alta complexidade para o estado e possui 17 unidades assistenciais.

\section{Participantes da pesquisa}

Para a realização da pesquisa, foi utilizado um instrumento estruturado composto por uma lista de indicadores elaborada com base no Guia de Recomendações de Anotação de Enfermagem do COFEN, publicado em 2016(5). Os indicadores escolhidos foram: registro do nome completo do paciente; informação do número do prontuário; data e hora do registro; presença de assinatura, categoria profissional e número de inscrição no respectivo Conselho Regional de Enfermagem (COREN); presença de rasuras; uso de letra legível: preenchimento completo dos impressos; frequência diária da evolução; utilização apenas de formulário padronizado pela instituição; presença de diagnóstico e prescrição de enfermagem; checagem da prescrição médica e de enfermagem conforme padronização do serviço; justificativa para não checagem da prescrição médica e de enfermagem; presença do histórico de Enfermagem; protocolos de prevenção de lesão por pressão e de queda. 


\section{Coleta de dados}

Para a coleta dos dados foi realizada entre os meses de setembro de 2017 e maio de 2018 mediante a seguinte estratégia: cada uma das 17 unidades assistenciais do hospital foi visitada por três dias consecutivos. Durante as visitas, todos os prontuários disponíveis no momento da coleta foram analisados de acordo com a lista de indicadores elaborada. No total, foram analisados 203 prontuários. Considerandose que o hospital possui 214 leitos ativos, foram analisados $94,8 \%$ dos prontuários.

\section{Procedimentos de análise dos dados}

Após a coleta dos dados, procedeu-se à digitação do banco de dados em uma planilha Excel. Em seguida, foi feita a análise estatística segundo frequência absoluta e relativa dos indicadores coletados.

\section{Procedimentos éticos}

O protocolo da pesquisa foi aprovado por um Comitê de Ética em Pesquisa, mediante a emissão do CAAE 66018217.8.0000.0049 e atendeu às recomendações éticas da Resolução 466/12 do Conselho Nacional de Saúde.

\section{RESULTADOS}

Foram analisados 203 prontuários de pacientes que estavam hospitalizados nas 17 unidades assistenciais do $\mathrm{HU}$.

Tabela 1: Indicadores de qualidade dos registros de enfermagem no prontuário. Salvador, Bahia, Brasil. 2018.

\begin{tabular}{|c|c|c|c|c|}
\hline Indicadores & Sim & $\%$ & Não & $\%$ \\
\hline Nome completo do paciente & 190 & $94 \%$ & 13 & $6 \%$ \\
\hline Número do prontuário & 188 & $93 \%$ & 15 & $7 \%$ \\
\hline Data e hora & 186 & $92 \%$ & 17 & $8 \%$ \\
\hline Presença de rasura & 68 & $33 \%$ & 135 & $67 \%$ \\
\hline Letra legivel & 184 & $91 \%$ & 19 & $9 \%$ \\
\hline Assinatura do profissional & 191 & $94 \%$ & 12 & $6 \%$ \\
\hline $\begin{array}{c}\text { Número do COREN junto à } \\
\text { assinatura }\end{array}$ & 138 & $68 \%$ & 65 & $32 \%$ \\
\hline $\begin{array}{c}\text { Informação da categoria } \\
\text { profissional }\end{array}$ & 60 & $30 \%$ & 143 & $70 \%$ \\
\hline Histórico de enfermagem & 186 & $92 \%$ & 17 & $8 \%$ \\
\hline Diagnóstico de enfermagem & 199 & $98 \%$ & 17 & $8 \%$ \\
\hline Prescrição de enfermagem & 199 & $98 \%$ & 4 & $2 \%$ \\
\hline $\begin{array}{l}\text { Checagem da prescrição } \\
\text { de enfermagem conforme } \\
\text { padronização da instituição }\end{array}$ & 133 & $66 \%$ & 70 & $34 \%$ \\
\hline $\begin{array}{l}\text { Justificativa para a não } \\
\text { checagem da prescrição de } \\
\text { enfermagem }\end{array}$ & 52 & $26 \%$ & 109 & $54 \%$ \\
\hline
\end{tabular}

\begin{tabular}{ccccc}
$\begin{array}{c}\text { Checagem da prescrição } \\
\text { médica conforme padronização } \\
\text { da instituição }\end{array}$ & 183 & $90 \%$ & 20 & $10 \%$ \\
$\begin{array}{c}\text { Justificativa para a não } \\
\text { checagem da prescrição médica }\end{array}$ & 45 & $22 \%$ & 116 & $57 \%$ \\
\hline $\begin{array}{c}\text { Evolução de enfermagem } \\
\text { Frequência diária da evolução }\end{array}$ & 199 & $98 \%$ & 4 & $2 \%$ \\
\hline $\begin{array}{c}\text { Registro dos controles dos } \\
\text { sinais vitais }\end{array}$ & 195 & $96 \%$ & 8 & $4 \%$ \\
$\begin{array}{c}\text { Avaliação do risco de lesão por } \\
\text { pressão - Escala de Braden }\end{array}$ & 123 & $61 \%$ & 80 & $39 \%$ \\
$\begin{array}{c}\text { Escala de risco de queda - } \\
\text { escala de Morse }\end{array}$ & 138 & $68 \%$ & 65 & $32 \%$ \\
\hline
\end{tabular}

Fonte: Coleta de dados da pesquisa.

Conforme pode ser observado na Tabela 1, nenhum dos indicadores auditados atingiu 100\% de conformidade. Os itens nome completo do paciente, presença do número do prontuário, data e hora do registro, utilização de letra legivel, assinatura do profissional responsável pelo registro; histórico, prescrição e evolução de enfermagem; registro dos controles dos sinais vitais dos pacientes; e checagem da prescrição médica conforme a padronização do hospital obtiveram percentuais de conformidade entre $90 \%$ e $98 \%$. É importante destacar que em 68\% dos prontuários não havia avaliação para o risco de queda e em 61\%, não se encontrou avaliação do risco de desenvolver lesão por pressão.

Por outro lado, foram identificadas rasuras em 33\% dos prontuários; em $70 \%$ dos registros não havia informação sobre a categoria profissional; e em $57 \%$ das prescrições de enfermagem e 54\% das prescrições médicas não checadas, não se encontrou justificativas para a não checagem.

\section{DISCUSSÃO}

A auditoria dos prontuários aponta para a necessidade de valorização da qualidade dos registros de enfermagem na prática, apesar dos altos índices de conformidade encontrados em alguns indicadores. Convergindo com outro estudo realizado no Brasil, o qual identificou um percentual de $96 \%$ de conformidade na identificação dos pacientes, ${ }^{(7)}$ a pesquisa revela que dados imprescindíveis à identificação de todos os campos dos impressos, como nome completo do paciente, número do prontuário, data e hora do registro obtiverem um elevado percentual de preenchimento adequado. Embora se entenda que seja uma responsabilidade da equipe de enfermagem realizar registros claros e fidedignos, que reflitam a assistência prestada aos pacientes, ${ }^{\left({ }^{(8)}\right.}$ essa realidade não foi evidenciada em todos os indicadores analisados.

A auditoria detalhada dos registros de enfermagem possibilita a identificação de possíveis deficiências da equipe na documentação da assistência prestada. De acordo com as normas contidas no Guia de Recomendações de Anotação de 
Enfermagem do COFEN, o registro correto, com data, hora e assinatura, é uma responsabilidade ética da enfermagem, normatizada por legislação específica ${ }^{(5)}$.

Os registros de enfermagem correspondem a cerca de $50 \%$ das informações sobre os cuidados registrados nos prontuários. Assim, destaca-se o seu papel central na comunicação entre a equipe multiprofissional, uma vez que geram informações que subsidiam a conduta de outros profissionais, facilitam o planejamento, a tomada de decisão e a continuidade do cuidado. Acrescenta-se também sua importância para a realização de auditorias, pesquisas, processos judiciais e planejamento. Como tal, é um elemento imprescindivel no processo de cuidado(9)

Alguns itens relacionados à identificação do profissional responsável pelo registro, como utilização de letra legível e assinatura, também obtiveram percentual de conformidade acima de $90 \%$. Por outro lado, a identificação da categoria profissional estava presente em apenas 30\%; e a informação do número do registro no COREN foi citada em apenas $68 \%$ dos prontuários. Essa informação vai de encontro à recomendação do COFEN, que estabelece a obrigatoriedade da identificação do profissional em todos os registros, além do preenchimento correto e de forma legivel, de todos os campos necessários à identificação do paciente (5). Problemas como letras ilegiveis podem resultar em dificuldades de entendimento entre a equipe, podendo levar a múltiplas interpretações e à ocorrência de erros ${ }^{(4)}$.

A deficiência na identificação do profissional também foi apontada em outros estudos, a exemplo de uma pesquisa realizada no estado de São Paulo, que revelou a ausência da identificação do profissional responsável pelo registro na maioria dos prontuários. ${ }^{(10)}$ Outra pesquisa realizada em um hospital universitário em Curitiba - PR, identificou que 22\% do total de registros analisados não continha a identificação do profissional responsável( ${ }^{(9)}$. Acredita-se que a Resolução do COFEN 0545/2017, que trata da obrigatoriedade do uso do carimbo pelos profissionais de enfermagem, poderá contribuir para melhorar a identificação correta do profissional(6).

Os achados indicam um elevado percentual de utilização de impressos próprios da Sistematização da Assistência de Enfermagem (SAE), como histórico, diagnóstico, prescrição e evolução. Este fato pode estar relacionado a uma fase mais avançada de implementação e sedimentação da SAE no campo da pesquisa. Outro estudo(10) indica um percentual de preenchimento do histórico de enfermagem em 99,9\%; e $88,4 \%$, para a prescrição de enfermagem. Já o diagnóstico de enfermagem alcançou não conformidade em 58,7\% e a evolução, em 64,6\%, no estudo citado. Destaca-se que a SAE desempenha um papel fundamental em descrever claramente as contribuições da Enfermagem no cenário de cuidados à saúde, favorecendo um atendimento organizado, ético e baseado em evidências científicas ${ }^{(11)}$

É importante ressaltar que há uma preocupação da equipe de enfermagem em checar corretamente os itens da prescrição médica (90\%), porém o registro das justificativas para as não checagens estava presente em apenas $22 \%$ dos prontuários. De maneira geral, observa-se itens da prescrição não administrados, em branco ou apenas circulado, sem, contudo, haver uma correlação quanto às justificativas para o não cumprimento, no impresso de anotações de enfermagem.

Por outro lado, chama a atenção o fato de que em apenas 66\% dos prontuários, a prescrição de enfermagem foi checada conforme a padronização da instituição. Este dado é $24 \%$ menor do que o índice da checagem correta da prescrição médica. Quando se considera as justificativas para a não checagem dos itens da prescrição de enfermagem, encontram-se dados convergentes com o mesmo item da prescrição médica. Estes dados podem estar relacionados a uma cultura interna que compreende e aceita apenas a presença do círculo em volta dos horários/turnos de aprazamento, sem uma cobrança efetiva do registro textual das justificativas. Assim, fica exposta uma possibilidade de quebra no fluxo de informações entre a equipe de enfermagem e multiprofissional, uma vez que a compreensão de todos os acontecimentos relacionados à assistência e evolução dos pacientes pode estar se dando em nível subjetivo, sem uma definição clara de regras que devem ser observadas e seguidas por todos os membros.

Itens relacionados a protocolos de segurança do paciente preconizados pela Agência Nacional de Vigilância Sanitária (ANVISA)(12) como avaliação do risco de queda, feita com a utilização da escala de Morse; e a avalição do risco para lesão por pressão, feita com a utilização da escala de Braden, estavam presentes em $68 \%$ e $61 \%$, respectivamente. Outras pesquisas também sinalizam a importância do fortalecimento da avaliação de riscos para garantia da segurança dos pacientes $^{(13,14)}$. No que diz respeito à avaliação do risco de queda entre os pacientes internados em regime integral, a política institucional reconhece a presença de risco intrínseco naqueles pacientes de 0 a 12 anos, acima de 60 anos, psiquiátricos, em preparo para colonoscopia e com osteogênese imperfeita. Assim, diante desse reconhecimento, não é preconizada o preenchimento do impresso de avaliação.

Estas informações, entretanto, indicam a necessidade de fortalecimento da cultura de segurança do paciente eum maior entendimento, aceitação e avaliação dos riscos a que estão expostos os pacientes hospitalizados, acrescidos dos riscos relacionados à própria doença. A auditoria destaca-se por sua importância como ferramenta que possibilita a identificação de problemas nos prontuários. Os dados produzidos pelos 
relatórios podem nortear a conduta da equipe no sentido de fornecer subsídios para práticas educativas, além de subsidiar outras ações inerentes à prática da enfermagem $(4,15)$. Salienta-se a importância de um trabalho de educação permanente junto à equipe que enfatize tanto a segurança do profissional, por meio da realização de registros corretos, fidedignos e válidos, quanto a segurança do paciente, com uma avaliação completa e que garanta o garantia do fluxo de informações entre a equipe multiprofissional.

No campo da pesquisa já vem sendo realizada uma ação desde o ano de 2017, utilizando-se de diversas estratégias como discussão do tema em sessões científicas, divulgação de material impresso nos murais, publicação de notas na rede interna de computadores e abordagem direta dos profissionais de enfermagem nos locais de trabalho(16).

Acredita-se que o prontuário seja um local de centralização e integração das informações sobre a evolução clínica dos pacientes. Como tal, defende-se a ideia de que, de acordo com os resultados da pesquisa, receba mais atenção por parte da equipe de enfermagem e multiprofissionais indo além da avaliação da forma dos registros.

\section{LIMITAÇÕES DA PESQUISA}

Esta pesquisa traz como limitações o fato de ter se atido apenas à forma dos registros, sem avaliar o conteúdo do que foi registrado pela enfermagem, cuja importância já vem sendo destacada por outros autores ${ }^{(4,8-10,15-17)}$. Entretanto, defendese que ambos os aspectos são importantes para a validação dos registros e que eles sejam complementares. Em todos os casos, quando realizado conforme as recomendações, indicam qualidade no cuidado de enfermagem e contribuem para uma maior visibilidade da profissão.

\section{CONCLUSÃO}

De acordo com os resultados obtidos, a qualidade dos registros de enfermagem, apesar de elevados percentuais de conformidade, atende parcialmente às recomendações do COFEN, devido à deficiência na identificação dos profissionais, nas justificativas das prescrições tanto de enfermagem, quanto médicas e presença de rasuras em registros. Os resultados sugerem a necessidade constante de atuação dos programas de educação permanente para a implementação de ações que visem à realização de registro de enfermagem com excelência.

A construção de melhores práticas assistenciais, além de programação de ações que visem melhorias nos resultados podem favorecer a continuidade do trabalho e garantir o fluxo de informações entre a equipe. Nesse interim, os registros podem contribuir para a melhoria da qualidade e da segurança do paciente e da equipe.

Este artigo está vinculado ao projeto de pesquisa Repercussões das capacitações de profissionais de enfermagem nas práticas assistenciais em um hospital universitário. financiado com uma bolsa de iniciação à pesquisa pelo Programa Permanecer edição 2017/2018, da Universidade Federal da Bahia (UFBA).

\section{Contribuição dos autores:}

Valdenir Almeida da Silva - concepção, desenho do estudo, análise e interpretação dos dados, revisão crítica, revisão final. Rosana Santos Mota - concepção, desenho do estudo, análise e interpretação dos dados, revisão crítica, revisão final.Larissa Silva Oliveira - coleta de dado, análise e interpretação dos dados. Nilcea de Jesus - coleta de dado, análise e interpretação dos dados.Cristiane Marques de Carvalho - análise e interpretação dos dados, redação do artigo.Lívia Gomes da Silva Magalhães - análise e interpretação dos dados, redação do artigo.

\section{Declaração de conflito de interesse:}

Os autores declaram não haver conflito de interesse. 


\section{REFERÊNCIAS}

1. Silva MA, Vieira ETV. Auditoria Interna: uma ferramenta de gestão dentro das organizações. Redeca FEA-PUC [Internet]. 2015 [cited 2018 Out 29]; 2(2):1-20. Available from: https://revistas.pucsp.br/redeca/article/view/28559/20046

2. Brasil. Auditoria do SUS no contexto do SNA: Qualificação do Relatório de Auditoria [Internet]. 2015 [cited 2018 Out 29]: 8-17. Available from: http://bvsms.saude.gov.br/bvs/publicacoes/auditoria_sus_contexto_sna.pdf

3. Vieira RQ, Sanna MC. Auditoria de enfermagem em periódicos cientificos internacionais no periodo de 1955-1972. Rev Enferm UFSM [Internet]. 2013 [cited 2018 Out 29]; 3(3): 528-538. Available from: https://periodicos.ufsm.br/reufsm/article/view/7511

4. Lira MAM. Avaliação da qualidade dos registros de enfermagem nos prontuários da clínica cirúrgica do Hospital Municipal de Brumado/BA por meio da auditoria. Revista Cientifica Multidisciplinar Núcleo do Conhecimento [Internet]. 2017 [cited 2018 Out 29]; 02,03(9): 24-36. Available from: https://www.nucleodoconhecimento.com.br/saude/avaliacao-da-qualidade

5. Conselho Federal de Enfermagem (COFEN). Guia de Recomendações para Registro de Enfermagem no Prontuário do Paciente e outros Documentos de Enfermagem. Portaria n. 523/2015 [Internet]. 2016 [cited 2018 Out 29]. Available from: http://www. cofen.gov.br/wp-content/uploads/2016/08/Guia-de-Recomenda\%C3\%A7\%C3\%B5es-CTLN-Vers\%C3\%A3o-Web.pdf

6. Conselho Federal de Enfermagem (COFEN). Resolução COFEN № 0564/2017. Aprova o novo Código de Ética dos Profissionais de Enfermagem [Internet]. 2017 [cited 2018 Out 29]. Available from: http://www.cofen.gov.br/resolucao-cofen-no-5642017_59145. html

7. Macedo MMCS, Almeida AF, Assad LG, Rocha RG, Ribeiro GSR, Pereira LMV. Identificación del paciente mediante pulsera electrónica en una unidad de terapia intensiva general de adultos. Revista de Enfermagem Referência [Internet]. 2017 [cited 2018 Out 29]; serIV(13), 63-70. Available from: http://www.scielo.mec.pt/scielo. php?script=sci_arttext\&pid=S087402832017000200007\&lng=pt.

8. NomuraATG, Barragan MS,Almeida MA. Quality ofnursing documentation before and after the Hospital Accreditation in a university hospital. Rev. Latino-Am. Enfermagem [Internet]. 2016 [cited 2018 Out 29];24:e2813. Available from: http://www.scielo.br/scielo.php?script=sci_arttext\&pid=S010411692016000100422\&lng=en.

9. Gomes DC, Cubas MR, Pleis LE, Shmeil, MAH, Peluci APVD. Termos utilizados por enfermeiros em registros de evolução do paciente. Rev Gaúcha Enferm [Internet]. 2016 [cited 2018 Out 29]; 37(1):e53927. Available from: http://www.scielo.br/scielo.php?script=sci_arttextSpid=S198314472016000100412\&lng=en.
10. Franco MTG, Akemi EN, D'Inocento M. Evaluation of the nursing records in the medical records of patients hospitalized in an internal medicine unit. Acta Paul Enferm [Internet]. 2012 [cited 2018 Out 29];25(2):163-70. Available from: http://www.scielo.br/scielo. php?script=sci_arttext\&pid=S0103210020120002000028lng=en.

11. Terças ACP, Souza IG, Moura ACP, Nascimento VF, Hattori TY, Gleriano JS, et al. Sistematização da Assistência de Enfermagem no monitoramento clinico de pacientes com hantavirose. Journal Health NPEPS [Internet]. 2017 [cited 2018 Out 29]; 2(2):391406. Available from: https://periodicos.unemat.br/index.php/ jhnpeps/article/view/1764

12. Agência Nacional de Vigilância Sanitária (ANVISA). Assistência Segura: Uma ReflexãoTeórica Aplicada à Prática [Internet]. 2013 [cited 2018 Out 29]. Available from: http://portal. anvisa.gov.br/documents/33852/3507912/Caderno+1++Assist\%C3\%AAncia+Segura++Uma+Reflex\%C3\%A3o+Te\%C3\%B3rica+Aplicada+\% C3\%A0+Pr\%C3\%Altica/978 $1798-$ cea0-4974-9d9b-077528eal573

13. Oliveira JLC, Silva SV, Santos PR, Matsuda LM, Tonini NS, Nicola AL. Patient safety: knowledge between multiprofessional residents. Einstein [Internet]. 2017 [cited 2018 Out 29];15(1):50-7. Available from: http://www.scielo.br/scielo.php?script=sci_arttext\&pid=S167945082017000100050\&lng=en.

14. Cedraz RO, Gallasch $\mathrm{CH}$, Pérez Júnior EF, Gomes FH, Rocha FG, Mininel VA. Gerenciamento de riscos em ambiente hospitalar: incidência e fatores de riscos associados à queda e lesão por pressão em unidade clinica. Esc Anna Nery [Internet]. 2018 [cited 2018 Out 29];22(1):e20170252. Available from: http:// www.scielo.br/pdf/ean/v22nl/pt_1414-8145-ean-2177-9465-EAN-2017-0252.pdf

15. Padilha EF, Haddad MCFL, Matsuda LM. Qualidade dos registros de enfermagem em terapia intensiva: avaliação por meio da auditoria retrospectiva. Cogitare Enferm [Internet]. 2014 [cited 2018 Out 29]; 19(2):239-45. Available from: https://revistas.ufpr. $\mathrm{br} /$ cogitare/article/view/32103

16. Silva VA, Mota RS, Mendes AS, Costa JCB, Magalhães LGS, Souza LL. Registro correto como segurança para o paciente e para a enfermagem. Enfermagem Brasil [Internet]. 2018 [cited 2018 Out 29];17(4):411-417. Available from: https://portalatlanticaeditora.com.br/index.php/enfermagembrasil/article/ view/2277/3893

17. Aquino MJN, Cavalcante TMC, Abreu RNDC, Scopacasa LF, Negreiros FDS. Anotações de enfermagem: avaliação da qualidade em Unidade de Terapia Intensiva. Enferm. Foco [Internet]. 2018 [cited 2018 Out 29]; 9 (1): 07-12. Available from: http://revista.cofen.gov.br/index.php/enfermagem/article/view/1314 